\title{
Histone Modifications Regulate the Developmental Expression of Human Hepatic UDP-Glucuronosyltransferase $1 \mathrm{~A} 1^{\mathrm{S}}$
}

\author{
Ya-Li Nie, Xiang-Guang Meng, Jing-Yang Liu, Liang Yan, Pei Wang, Hong-Zheng Bi, \\ Quan-Cheng Kan, and Li-Rong Zhang
}

\begin{abstract}
Department of Pharmacology, School of Basic Medicine, Zhengzhou University (Y.-L.N., J.-Y.L., L.Y., P.W., H.-Z.B., L.-R.Z.); Laboratory of Cardiovascular Disease and Drug Research, Seventh People's Hospital of Zhengzhou (X.-G.M.); Department of Clinical Pharmacology, First Affiliated Hospital, Zhengzhou University (Q.-C.K.), Zhengzhou, People's Republic of China
\end{abstract}

Received April 14, 2017; accepted September 29, 2017

\section{ABSTRACT}

Human UDP-glucuronosyltransferase 1A1 (UGT1A1) is a unique enzyme involved in bilirubin conjugation. We previously characterized the hepatic expression of transcription factors affecting UGT1A1 expression during development. Accordingly, in this study, we characterized the ontogenetic expression of hepatic UGT1A1 from the perspective of epigenetic regulation. We observed significant histone-3-lysine-4 dimethylation (H3K4me2) enrichment in the adult liver and histone-3-lysine-27 trimethylation (H3K27me3) enrichment in the fetal liver, indicating that dynamic alterations of histone methylation were associated with ontogenetic UGT1A1 expression. We further showed that the transcription factor hepatocyte nuclear factor $1 \alpha$ (HNF1A) affects histone modifications around the UGT1A1 locus. In particular, we demonstrated that by recruiting HNF1A the cofactors mixed-lineage leukemia 1, the transcriptional coactivator $\mathrm{p} 300$, and nuclear receptor coactivator 6 aggregate at the UGT1A1 promoter, thereby regulating histone modifications and subsequent UGT1A1 expression. In this study, we proposed new ideas for the developmental regulation of metabolic enzymes via histone modifications, and our findings will potentially contribute to the development of age-specific therapies.

\section{Introduction}

Human UDP-glucuronosyltransferase 1A1 (UGT1A1) is a unique enzyme involved in bilirubin conjugation, which prevents hyperbilirubinemia and jaundice (Bosma et al., 1994; Kanou et al., 2004). Previous reports showed that hepatic UGT1A1-expression levels exhibit a dynamic pattern during development and maturation. Briefly, UGT1A1 is highly expressed in adult livers but is present at very low levels, or even absent, in fetal livers (Kawade and Onishi, 1981; Coughtrie et al., 1988; Burchell et al., 1989; Miyagi and Collier, 2011; Nie et al., 2017). Although the DNA sequence of the UGT1Al gene in human hepatocytes is identical, there are obvious differences in UGT1A1 expression from the prenatal to adult stages. Thus, variation in UGT1A1 expression during human hepatic development is not entirely explained by the genome, but is closely related to the epigenome.

Epigenetic mechanisms, which mainly include DNA methylation, histone modifications, and noncoding RNA molecules, generate reversible but heritable changes in gene expression without alteration of nuclear DNA sequences (Egger et al., 2004; Bird, 2007). Increasing experimental evidence indicates that, in the regulation of gene

This work was supported by the National Natural Science Foundation of China [Grants 81173127, U1604163, and U1404833].

https://doi.org/10.1124/dmd.117.076109.

S This article has supplemental material available at dmd.aspetjournals.org. expression, the methylation and acetylation of histones $\mathrm{H} 3$ and $\mathrm{H} 4$ are the most common and functionally important modifications (Ivanov et al., 2014). Histone methylation can activate gene expression or inhibit gene transcription (Xiao et al., 2003; Shi et al., 2006, 2007; Wysocka et al., 2006), and histone acetylation promotes gene expression, whereas deacetylation inhibits gene expression (Peng and Zhong, 2015; Di Martile et al., 2016).

Furthermore, epigenetic regulation is important for drugmetabolizing enzymes (Ingelman-Sundberg et al., 2013; Kim et al., 2014; Zanger et al., 2014; Peng and Zhong, 2015). As for phase II enzymes, the epigenetic mechanisms contributing to UGT1A1 regulation have been reported. For example, differential DNA methylation within the UGT1A1 promoter region may in part be responsible for interindividual variations in UGT1A1 expression and activity (Yasar et al., 2013). In addition, tissue-specific UGT1A1 levels are associated with DNA hypermethylation. Along with histone H3 hypoacetylation, DNA hypermethylation diminishes hepatocyte nuclear factor $1 \alpha(\mathrm{HNF} 1 \mathrm{~A})$ binding, further contributing to inhibition of UGT1A1 expression in human kidney (Oda et al., 2013). DNA methylation also leads to silencing of UGT1A1 expression in colon cancer (Gagnon et al., 2006). Additionally, microRNA-491-3p is a key regulatory factor of UGT1A1 expression and activity (Dluzen et al., 2014). However, the role of epigenetic modifications in ontogenetic UGT1A1 regulation remains unclear. This study explored the histone modifications involved in regulating human hepatic UGT1A1 expression during development.

ABBREVIATIONS: ChIP, chromatin immunoprecipitation; ChIP-seq, ChIP sequencing; co-IP, coimmunoprecipitation; EZH2, enhancer of zeste homolog 2; GAPDH, glyceraldehyde-3-phosphate dehydrogenase; H3K4me2, histone-3-lysine-4 dimethylation; H3K27me3, histone-3-lysine-27 trimethylation; HNF1A, hepatocyte nuclear factor $1 \alpha$; HNF4A, hepatocyte nuclear factor $4 \alpha$; HNF1AE, HNF1A response element; MLL1, mixed lineage leukemia 1; NCOA6, nuclear receptor coactivator 6; qRT-PCR, quantitative real-time polymerase chain reaction; siRNA, small interfering RNA; UGT1A1, UDP-glucuronosyltransferase 1 A1. 


\section{Materials and Methods}

Liver Sample Collection and Ethics Statement. All livers donor (30 fetuses and 30 adults) were from the First Affiliated Hospital of Zhengzhou University in a Han Chinese population (detailed information is presented in Supplemental Table 1). Adult samples were derived from hepatectomy most often because of hepatic hemangioma. Prenatal samples were obtained from legal abortion. Written informed consent was obtained from each participant. Both adult donors and mothers of fetus donors exhibited normal liver function. This study was conducted in accordance with the Declaration of Helsinki and approved by the Ethics Committees of the First Affiliated Hospital of Zhengzhou University.

Cell Culture and Small Interfering RNA Transfection. HepG2 (a human hepatoma cell line) and LS174T (a human colon cell line) were cultured at $37^{\circ} \mathrm{C}$ under 5\% $\mathrm{CO}_{2}$ in Dulbecco's modified Eagle's medium with $10 \%$ fetal bovine serum. HNF1A small interfering RNA (siRNA) was synthesized by Invitrogen (Shanghai, People's Republic of China), with specific interference targets (5'-GCUAGUGGAGGAGUGCAAUTT-3' and 5'-AUUGCACUCCUCCACUAGCTT- $3^{\prime}$ ) and negative-control sequences (5'-UUCUCCGAACGUGUCACGUT- $3^{\prime}$ and 5'-ACGUGACACGUUCAGAGAATT-3'). HNF1A siRNA was transfected into both cell lines using Lipofectamine 2000 (Life Technologies, Carlsbad, CA) in six-well plates according to the manufacturer's instructions. To determine knockdown efficiencies, we collected cells at 24 and 48 hours after transfection for the measurement of mRNA and protein levels, respectively.

Quantitative Real Time Polymerase Chain Reaction. Total RNA was extracted using TRIzol reagent (Invitrogen, Carlsbad, CA) according to the manufacturer's protocols. We prepared cDNA from $1 \mu \mathrm{g}$ of total RNA using a PrimeScript RT reagent kit (Takara, Dalian, People's Republic of China). The relative mRNA levels of the genes of interest were measured by quantitative realtime polymerase chain reaction (qRT-PCR) and normalized to those of glyceraldehyde-3-phosphate dehydrogenase $(G A P D H)$ mRNA levels using SYBR Premix Ex Taq II (Takara). Sequences of the primers used for qRT-PCR are presented in Supplemental Table 2.

Western Blot Analysis. Total proteins collected from cell lines at 48 hours after transfection were prepared using radioimmunoprecipitation assay buffer, and those from livers were isolated using total protein-extraction reagent for mammalian tissue (Boster Bio, Wuhan, People's Republic of China). Protein concentrations were determined using a bicinchoninic acid protein assay kit (ThermoFisher Scientific, Waltham, MA). Proteins $(50 \mu \mathrm{g})$ were separated by $6 \% \sim 12 \%$ SDS-polyacrylamide gel electrophoresis and transferred onto polyvinylidene fluoride membranes (Millipore, Billerica, MA). The relative protein levels of target genes were determined by immunoblotting using the anti-UGT1A1 antibody (ab170858; Abcam, Cambridge, United Kingdom), anti-HNF1A (ab174653; Abcam) antibody, and anti-enhancer of zeste homolog 2 (EZH2) antibody (21800-1-AP; Proteintech, Wuhan, People's Republic of China). The anti-GAPDH antibody (10494-1-AP; Proteintech) was used as an internal control for protein loading. All experiments were performed in duplicate.

Chromatin Immunoprecipitation Analysis. Chromatin immunoprecipitation (ChIP) assays were performed using the EZ-Magna ChIP HiSens ChIP kit (17-10461; Millipore). Briefly, liver tissue samples were cut into small pieces and incubated with $1 \%$ formaldehyde for 10 minutes at room temperature. Samples were then incubated in glycine for 5 minutes at room temperature to quench crosslinks. A Bioruptor (Pico; Diagenode, Liège, Belgium) was used for the sonication of nuclear lysates as follows: 45 seconds on and 30 seconds off for 25 cycles. The fragment size of sheared chromatin was determined by agarose gel electrophoresis with visualization using ethidium bromide. DNA fragments between 50 and 500 base pairs were immunoprecipitated using antibodies against demethylated histone-3-lysine-4 (H3K4me2; 17-677; Millipore) and trimethylated histone3-lysine-27 (H3K27me3; 17-622; Millipore). DNA was purified using a PCR purification kit (Qiagen, Hilden, Germany), and genomic DNA (input DNA) was prepared as a control.

The enriched DNA generated by ChIP from four samples (two fetuses and two adults) randomly selected from 10 cases (detailed information is presented in Supplemental Table 3) was sent to the Huada Company (Shenzhen, People's Republic of China) for high-throughput sequencing and bioinformatics analysis. Statistically significant ChIP-enriched peaks were identified by a comparison of the two groups of samples (fetal and adult livers) based on $P$ values. To validate the ChIP-sequencing (ChIP-seq) results, qPCR was performed using the enriched
DNA fragments generated by the ChIP assay as template. Primers are listed in Supplemental Table 4.

HepG2 and LS174T cells were collected 48 hours after transfection for ChIP analysis using specific antibodies targeting $\mathrm{H} 3 \mathrm{~K} 4 \mathrm{me} 2, \mathrm{H} 3$ acetylation (06-599; Millipore), nuclear receptor coactivator 6 (NCOA6; 25241-1-AP; Proteintech), and HNF1A to investigate histone modifications at a site (P1; Fig. 1B) located near the UGTIAl promoter. Enriched DNA fragments were collected for qPCR analysis. ChIP-qPCR data are plotted as percentages of input DNA.

Coimmunoprecipitation Assay. Coimmunoprecipitation (co-IP) of mixed lineage leukemia 1 (MLL1) and HNF1A, p300 and HNF1A, and NCOA6 and HNF1A was performed using Pierce Classic IP kits (26146; Thermo Fisher Scientific) according to the manufacturer's protocol. LS174T cells were harvested and lysed in the immunoprecipitation buffer. Using unprecipitated extracts as an input, whole-cell extracts were immunoprecipitated with anti-MLL1 (14689; CST, Danvers, MA), anti-NCOA6 (Proteintech), or anti-p300 (ab14984; Abcam), followed by immunoblotting assays performed with the anti-HNF1A antibody. Reciprocal immunoprecipitations were then performed using anti-HNF1A antibody, and Western blot analysis was performed with anti-MLL1, antiNCOA6, and anti-p300 antibodies.

Statistical Analysis. Data are expressed as mean \pm S.D. SPSS 19.0 (IBM, Armonk, NY) was used for statistical analysis. The statistical significance of differences between groups was compared using unpaired $t$ tests. Spearman's rank correlation analysis was also performed. Additionally, differences among ChIPseq results were evaluated by comparison of the two groups using $P$-values based on the Poisson distribution. $P<0.05$ was considered statistically significant.

\section{Results}

Ontogenetic Changes in UGT1A1 Expression Are Associated with Dynamic Alterations of Histone Methylation. H3K4me2 and H3K27me 3 profiles along the UGT1A1 locus in fetal and adult livers are summarized in Fig. 1. There was strong enrichment of H3K4me2 along a 5873 base pair sequence around the $U G T 1 A 1$ promoter in adult liver and enrichment of H3K27me3 12 kilobase upstream of the UGT1A1 locus in fetal liver. Our previous results suggested that UGT1A1 expression is very low in fetal livers; however, its expression level is significantly higher in adults (Nie et al., 2017). Hence, the observed enrichment of $\mathrm{H} 3 \mathrm{~K} 4 \mathrm{me} 2$ at UGT1A1 in adult liver was consistent with the higher UGT1A1 expression level at this developmental stage. Similarly, enrichment of $\mathrm{H} 3 \mathrm{~K} 27 \mathrm{me} 3$ at the UGT1A1 locus in fetal liver was consistent with the previous observations of low levels of UGT1A1 expression early in development. These data suggest that the histonemodification marker $\mathrm{H} 3 \mathrm{~K} 4 \mathrm{me} 2$ may explain the transcriptional activation of UGT1A1 in the adult liver, and that $\mathrm{H} 3 \mathrm{~K} 27 \mathrm{me} 3$, a marker of transcription inhibition, could silence the mRNA expression of UGT1A1 in the fetal liver.

Dynamic alterations of $\mathrm{H} 3 \mathrm{~K} 4 \mathrm{me} 2$ and $\mathrm{H} 3 \mathrm{~K} 27 \mathrm{me} 3$ along the UGT1A1 locus during liver development were verified by ChIPqPCR analysis (Fig. 1, C and D). Relative to fetal liver, H3K4me2 was enriched by an average of 3.2-fold in the adult liver, whereas compared with adult liver the average enrichment of H3K27me3 was 3.7-fold in the fetal liver. These ChIP-qPCR results are consistent with the findings from ChIP-seq analysis and confirm the reliability of the ChIP-seq data.

HNF1A siRNA Affects H3K4me2 and H3K27me3 Histone Modifications in the UGT1A1-Promoter Region in HepG2 and LS174T Cells. HNF1A knockdown in HepG2 and LS174T cells using siRNA caused a significant and specific decrease in corresponding mRNA and protein levels. Additionally, siRNA-treated cells exhibited relatively low UGTIA1 mRNA and protein expression compared with levels observed in controls (Fig. 2, A-C), and the differences were statistically significant $(P<0.01)$.

ChIP-seq results showed that the H3K4me2-enriched peak in the UGT1A1-promoter region in adult samples involved the HNF1A 
A

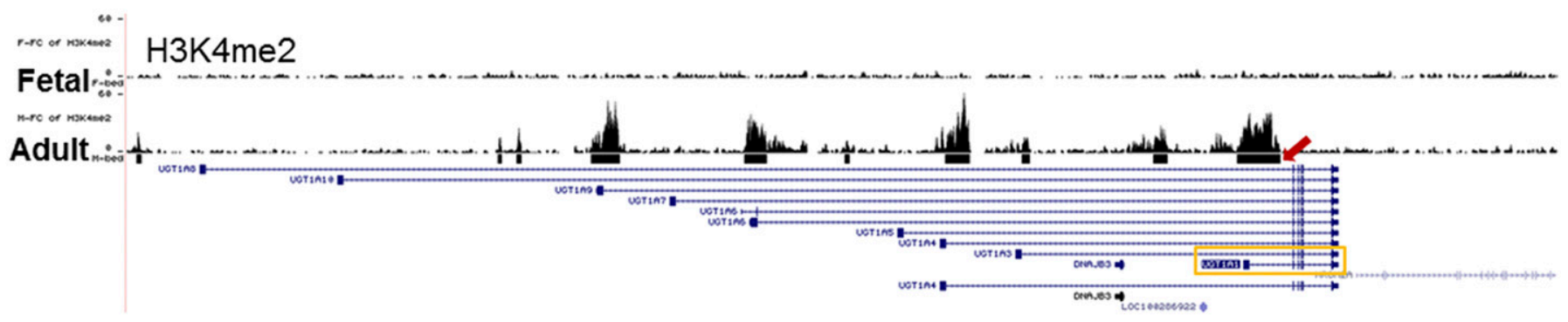

H3K27me3
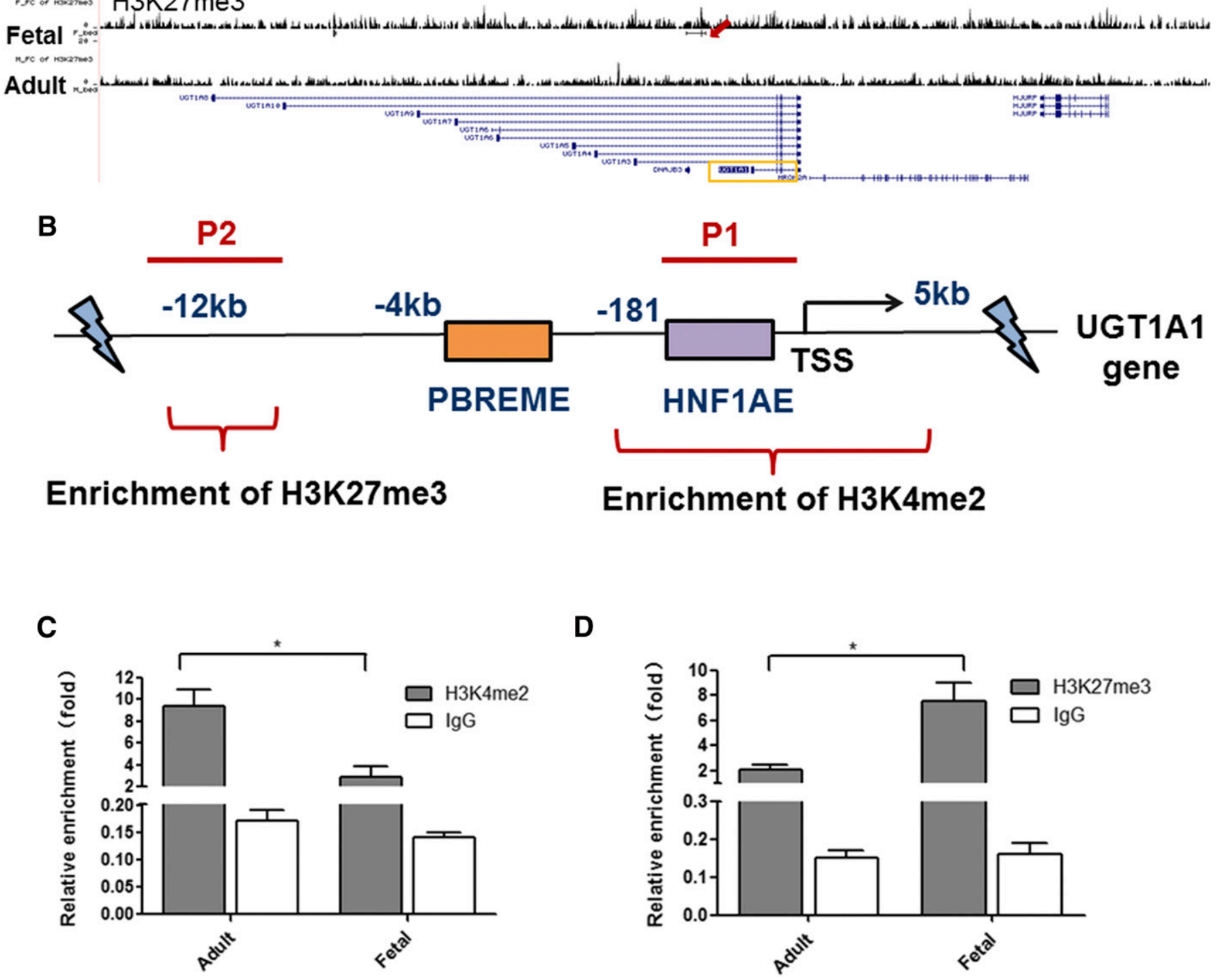

Fig. 1. Dynamic alterations of histone methylation were associated with the ontogenetic expression of UGTIA1. (A) H3K4me2 and H3K27me3 profiles along the UGTIAI locus in fetal and adult liver determined by ChIP-seq, presented using the UCSC genome browser. Red arrows represent enrichment sites; yellow boxes represent the UGT1A1 locus. (B) Schematic depiction of the UGT1A1 promoter $(-12,000$ to +5000$)$. Primer sets for amplification for ChIP-qPCR (P1-P2). TSS, transcription start site. (C) Validation of ChIP-seq results for H3K4me2 by ChIP-qPCR using primer pair 1 (P1). Average enrichment of H3K4me2 was 3.2-fold in adult livers as compared with fetal livers. (D) Validation of ChIP-seq results for H3K27me3 by ChIP-qPCR using primer pair 2 (P2). Average enrichment of H3K27me3 was 3.7-fold in fetal livers as compared with adult livers. Data presented are the mean \pm S.D. of three independent experiments. $* P<0.05, * * P<0.01$ vs. fetal livers.

response element (HNF1AE) (Fig. 1B). To evaluate the influence of HNF1A on histone modification of the region around the UGT1A1 promoter, HepG2 and LS174T cells were transfected with HNF1A siRNA, followed by ChIP analysis (Fig. 2, D and E). As expected, HNF1A knockdown caused a significant decrease in the enrichment of
$\mathrm{H} 3 \mathrm{~K} 4 \mathrm{me} 2$ and $\mathrm{H} 3$ acetylation around the HNF1AE in the UGT1A1promoter region. NCOA6 and HNF1A levels were also negatively affected by HNF1A knockdown in the same regions. These results suggest that knockdown of HNF1A significantly decreased UGT1A1 expression, which was accompanied by altered levels of $\mathrm{H} 3 \mathrm{~K} 4 \mathrm{me} 2$ and 

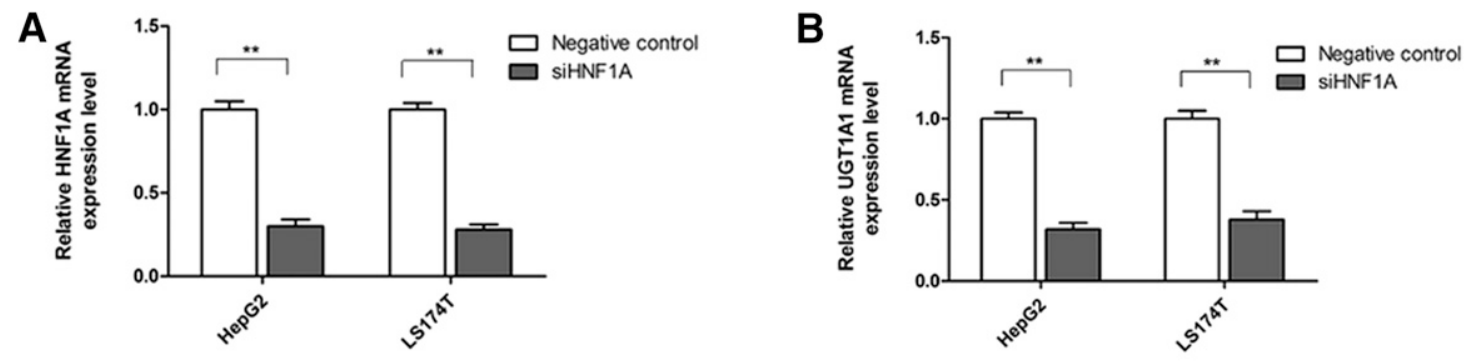

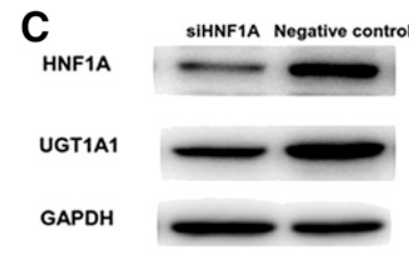

LS174T cell

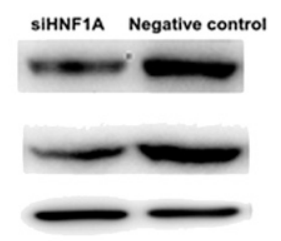

HepG2 cell

D

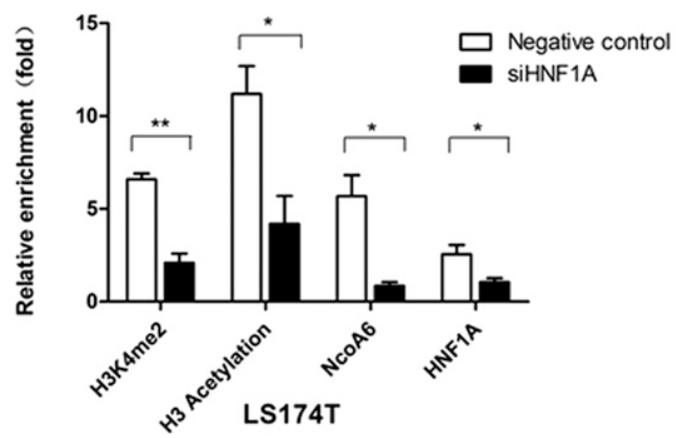

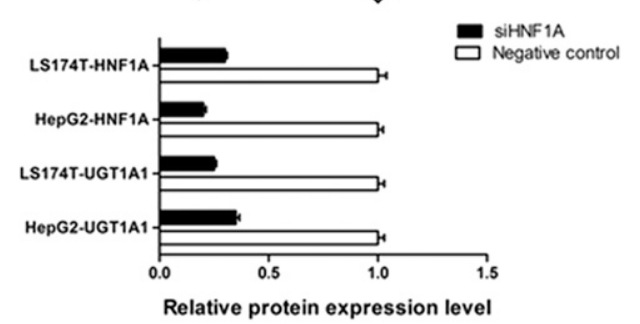

E

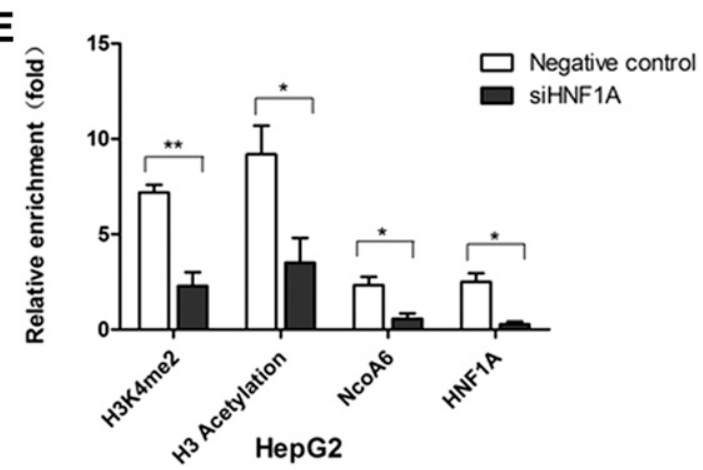

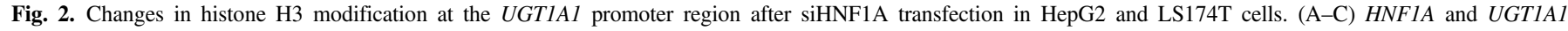

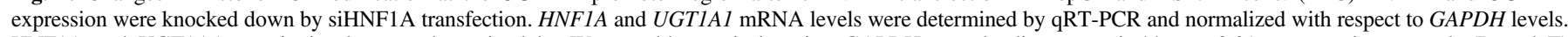

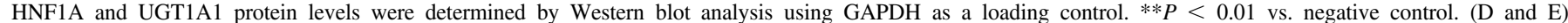

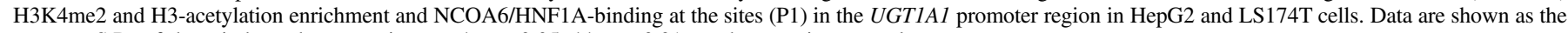
mean \pm S.D. of three independent experiments. $* P<0.05, * * P<0.01$ vs. the negative control.

$\mathrm{H} 3$ acetylation in the $U G T 1 A 1$-promoter region as well as reduced levels of NCOA6 and HNF1A expression.

MLL1/NCOA6/p300 Interact with HNF1A in LS174T Cells. The dimethylated state of histone-3-lysine-4 (H3K4me2) is achieved by selfrecruitment of the histone methyltransferase MLL1 (Rickels et al., 2016; Sun et al., 2016). Additionally, NCOA6, which is a ligand-dependent nuclear-receptor-interacting protein, accounts for a significant proportion of transcriptional coactivation (Mahajan and Samuels, 2008). Moreover, the transcriptional coactivator p300 can also act as a histone-acetyltransferase and contribute to transcriptional activation through its function in chromatin modification. Based on co-IP analysis (Fig. 3A), the histone-methylating enzyme MLL1 and the transcription factor HNF1A coexisted in a complex in LS174T cells, indicating an interaction between these two proteins. Similarly, NCOA6/p300 and HNF1A also coexisted in a complex (Fig. 3, B and C).

Correlation between EZH2 and UGT1A1 Expression in Human Liver. Generation of the trimethylated state of histone-3-lysine-27 (H3K27me3) is catalyzed by the histone methyltransferase EZH2 (Sharma et al., 2017). Our results indicate that EZH2 is expressed at high levels in fetal liver, with lower levels in adult liver samples (Fig. 4A). By contrast, UGT1A1 was expressed at significantly lower levels in fetal livers, with high levels observed in adult livers $(P<0.001)$ (Fig. 4B). According to Spearman's correlation analysis, UGT1A1 protein levels were negatively correlated with those of EZH2 $\left(r_{\mathrm{s}}=-0.74 ; P<0.001\right)$, and curve-fitting analysis showed that EZH2 protein levels may represent a power function of UGT1A1 protein levels (Fig. 4C).

\section{Discussion}

Accumulating evidence indicates that the epigenetic regulation of gene expression is essential for cell differentiation and organ development (Jaenisch and Bird, 2003; Sasaki and Matsui, 2008). Histone modifications of drug-metabolizing enzymes have been emphasized in recent years. In particular, $\mathrm{H} 3 \mathrm{~K} 4 \mathrm{me} 2$ and $\mathrm{H} 3 \mathrm{~K} 27 \mathrm{me} 3$ are involved in the ontogenetic expression of Cyp3a during mouse liver maturation ( $\mathrm{Li}$ et al., 2009b). Increases in H3K4me 2 can promote Cyp3a16 expression in the neonatal rat liver and Cyp3a11 expression in the adult mouse liver, whereas decreased $\mathrm{H} 3 \mathrm{~K} 4 \mathrm{me} 2$ and increased $\mathrm{H} 3 \mathrm{~K} 27 \mathrm{me} 3$ are associated with inhibition of Cyp3a16 expression in adult animals. Similarly, we previously characterized dynamic changes in H3K4me2 and H3K27me3 in the developmental switch of human hepatic CYP3A7 and CYP3A4 expression (He et al., 2016). Our present data demonstrate that $\mathrm{H} 3 \mathrm{~K} 4 \mathrm{me} 2$ enrichment in the adult liver correlates with UGT1A1 activation, whereas $\mathrm{H} 3 \mathrm{~K} 27 \mathrm{me} 3$ enrichment in the fetal liver could contribute to UGT1A1 suppression. Presumably, as a part of a developmental switch, the activation marker $\mathrm{H} 3 \mathrm{~K} 4 \mathrm{me} 2$ and the inhibitory marker H3K27me3 are involved in the dynamic expression of hepatic UGT1A1. Indeed, UGT1A1 protein levels in early fetal livers (20-30 weeks) are low compared with those of later fetal ( $>30$ weeks) 

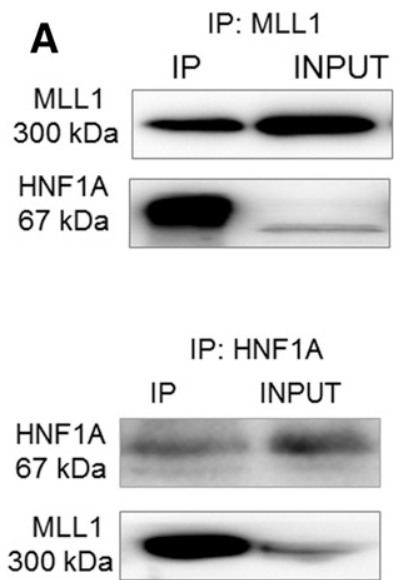
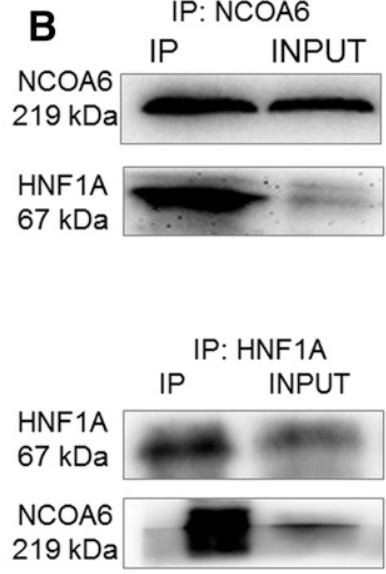

LS174T cell

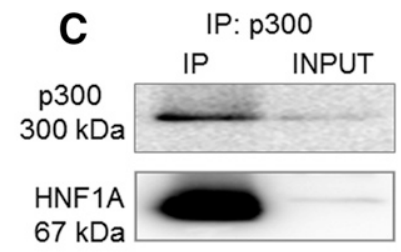

Fig. 3. Protein-protein interactions determined by co-IP assay in LS174T cells. (A) HNF1A interacts with MLL1. (B) HNF1A interacts with NCOA6. (C) HNF1A interacts with p300. Cell proteins before (input) and after (IP) immunoprecipitation were separated by SDSPAGE, transferred onto polyvinylidene fluoride membranes, and analyzed with the indicated antibodies. and neonatal livers, which is in agreement with previous reports. Hence, the results of the present study provide a rationale for the lower levels of UGT1A1 protein and activity in early fetal life; however, at later time points ( $>30$ weeks), fetal liver UGT1A1 protein levels are similar to or the same as those of neonates, yet there is negligible UGT1A1 enzyme activity. Further experiments are required to decipher the reasons for little-to-no activity of UGT1A1 in the later fetal livers.

Notably, our data confirm the enrichment of $\mathrm{H} 3 \mathrm{~K} 4 \mathrm{me} 2$ in the UGT1A1-promoter region from the transcription initiation site to approximately 5 kilobase downstream (i.e., the first exon) in the adult liver. Interestingly, the HNF1AE was included in the region of H3K4me 2 enrichment. Numerous studies have demonstrated that, as an evolutionarily conserved transcription factor, HNF1A can mediate the transcriptional activation of UGTs, including UGT1A1, 1A3, 1A4, 1A9, and UGT2B7 (Bernard et al., 1999; Ishii et al., 2000; Toide et al., 2002; Gregory et al., 2004; Odom et al., 2004; Gardner-Stephen and Mackenzie, 2007; Ramírez et al., 2008; Li et al., 2009a;
Ehmer et al., 2010). Additionally, H3K4me2 is generated by the histone methyltransferase MLL1; however, due to a lack of intrinsic DNA-binding activity, histone-modifying enzymes need to be recruited to genomic DNA by other factors, such as transcription factors. Histone-modifying enzymes then interact with transcription factors and are targeted to particular genomic loci via site-specific DNA-binding activity (Rosenfeld et al., 2006; Ivanov et al., 2014). Furthermore, co-IP assays provided evidence of an interaction between MLL1 and HNF1A; therefore, we speculate that by binding to the DNA-response element located near the UGT1Al-promoter region, HNF1A may recruit the histone-modifying enzyme MLL1 to promote the transcription of human hepatic UGT1A1.

Our data also indicate a potential interaction between HNF1A and NCOA6/p300. NCOA6 is known to interact with constitutive androstane receptor and hepatocyte nuclear factor $4 \alpha$ (HNF4A) as well as pregnane $\mathrm{X}$ receptor and HNF4A in the activation of CYP2C9 (Surapureddi et al., 2008, 2011). Moreover, HNF1A interacts with
A
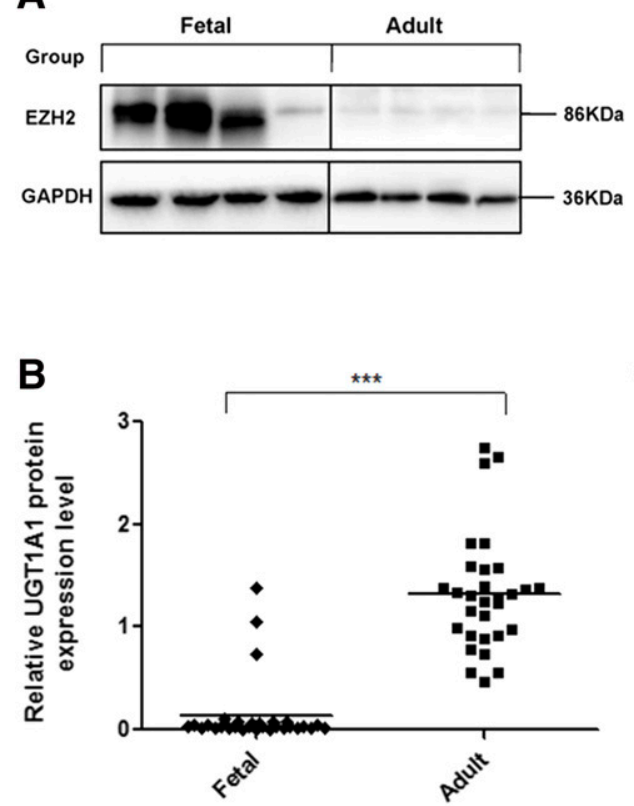
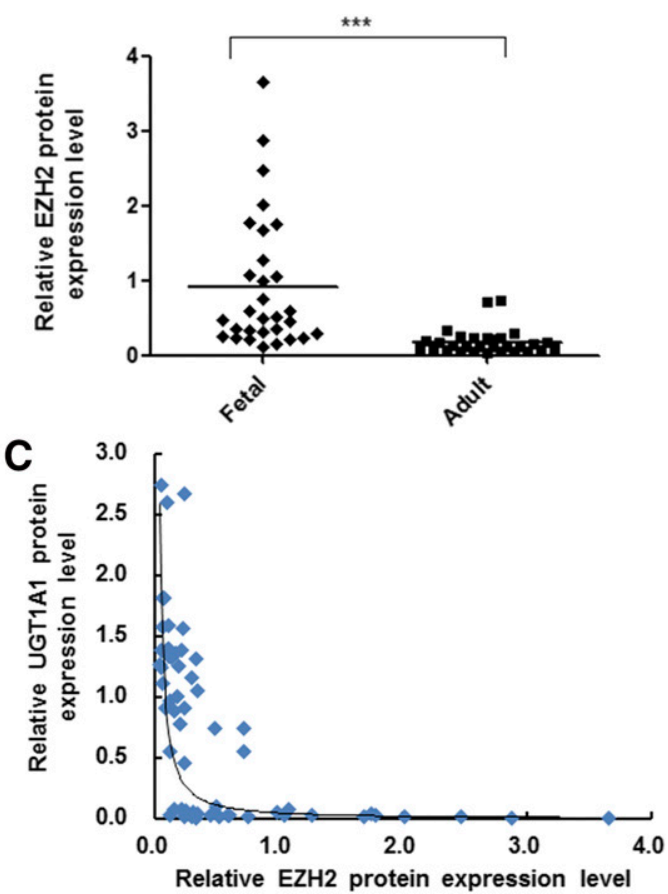

Fig. 4. Analyses of correlations between UGT1A1 and EZH2 protein levels in 60 human liver samples. (A) EZH2 expression was determined by Western blot analysis using GAPDH as a loading control. (B) UGT1A1 protein expression was measured by Western blot analysis. Data are expressed as mean \pm S.D., and groups were compared using unpaired $t$ tests. $* * * P<0.001$ vs. fetal liver samples. (C) The fitted curve showing the power function correlation between UGT1A1 protein levels and EZH2 expression. Differences were considered significant at $P<0.05$. 


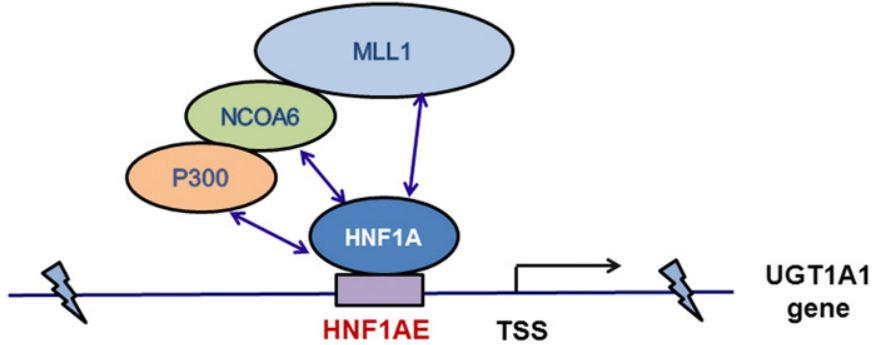

Fig. 5. Diagram of cofactors that interact with HNF1A in the UGTIA1 promoter.

p300 on the promoter of the glucose transporter GLUT2 (Ban et al., 2002). Additionally, our recent study reported that rifampicin enhances interactions between pregnane $\mathrm{X}$ receptor and NCOA6/p300 in the induction of CYP3A4 (Yan et al., 2017). Similarly, in the present study HNF1A interacted with $\mathrm{NCOA} 6 / \mathrm{p} 300$ to activate UGT1A1 expression. As expected, we demonstrated that HNF1A affected $\mathrm{H} 3$ acetylation as well as $\mathrm{H} 3 \mathrm{~K} 4 \mathrm{me} 2$ near the HNF1A-binding site in the UGT1A1 promoter. Therefore, our results clarified the mechanisms by which histone modifications regulate the expression of human hepatic UGT1A1. Briefly, the recruitment of HNF1A leads to aggregation of MLL1, NCOA6, and p300 at the UGT1A1-promoter region to regulate $\mathrm{H} 3 \mathrm{~K} 4 \mathrm{me} 2, \mathrm{H} 3$ acetylation, and subsequent $U G T 1 A 1$ expression (Fig. 5).

EZH2 is an important component of the Polycomb repressive complex, a specific methyltransferase of histone H3K27. Hence, EZH2 is crucial for catalyzing the $\mathrm{H} 3 \mathrm{~K} 27 \mathrm{me} 3$ epigenetic status and maintaining transcriptional silencing, particularly during embryonic development and cell differentiation (Otte and Kwaks, 2003; Lund and van Lohuizen, 2004). We observed a significant negative correlation between EZH2 and UGT1A1 levels; therefore, we speculate that high levels of EZH2 expression during embryonic development catalyze the formation of H3K27me3, leading to its enrichment in the fetal liver and thereby inhibiting UGT1A1 expression. Conversely, low levels of EZH2 expression in the adult liver release the transcriptional repression of UGT1A1. However, similar to MLL1 and as a histone-modifying enzyme, EZH2 lacks the ability to bind to specific DNA sequences; therefore, the roles of transcription factors and other cofactors involved in $\mathrm{EZH} 2$ recruitment and subsequent regulation of UGT1A1 expression require further investigation.

In conclusion, our results indicate that dynamic alterations of histone methylation are associated with ontogenetic UGTIAl expression. In particular, our findings suggest that the recruitment of HNF1A leads to aggregation of the cofactors MLL1, NCOA6, and p300 at the UGT1A1 promoter, which regulates histone modifications and subsequent UGT1A1 expression. These findings broaden our understanding of UGT1A1 expression during liver ontogeny, in the context of epigenetic regulation, and could potentially contribute to the development of agespecific therapies in pediatric patients.

\section{Authorship Contributions}

Participated in research design: Zhang, Kan, Nie.

Conducted experiments: Nie, Liu, Yan, Wang, Bi.

Performed data analysis: Nie, Meng.

Wrote or contributed to the writing of the manuscript: Nie, Zhang.

\section{References}

Ban N, Yamada Y, Someya Y, Miyawaki K, Ihara Y, Hosokawa M, Toyokuni S, Tsuda K, and Seino Y (2002) Hepatocyte nuclear factor- $1 \alpha$ recruits the transcriptional co-activator p300 on the GLUT2 gene promoter. Diabetes 51:1409-1418.

Bernard P, Goudonnet H, Artur Y, Desvergne B, and Wahli W (1999) Activation of the mouse TATA-less and human TATA-containing UDP-glucuronosyltransferase $1 \mathrm{~A} 1$ promoters by hepatocyte nuclear factor 1. Mol Pharmacol 56:526-536.
Bird A (2007) Perceptions of epigenetics. Nature 447:396-398.

Bosma PJ, Seppen J, Goldhoorn B, Bakker C, Oude Elferink RP, Chowdhury JR, Chowdhury NR, and Jansen PL (1994) Bilirubin UDP-glucuronosyltransferase 1 is the only relevant bilirubin glucuronidating isoform in man. J Biol Chem 269:17960-17964

Burchell B, Coughtrie M, Jackson M, Harding D, Fournel-Gigleux S, Leakey J, and Hume R (1989) Development of human liver UDP-glucuronosyltransferases. Dev Pharmacol Ther 13: $70-77$.

Coughtrie MW, Burchell B, Leakey JE, and Hume R (1988) The inadequacy of perinatal glucuronidation: immunoblot analysis of the developmental expression of individual UDPglucuronosyltransferase isoenzymes in rat and human liver microsomes. Mol Pharmacol 34: 729-735.

Di Martile M, Del Bufalo D, and Trisciuoglio D (2016) The multifaceted role of lysine acetylation in cancer: prognostic biomarker and therapeutic target. Oncotarget 7:55789-55810.

Dluzen DF, Sun D, Salzberg AC, Jones N, Bushey RT, Robertson GP, and Lazarus P (2014) Regulation of UDP-glucuronosyltransferase 1A1 expression and activity by microRNA 491-3p. $J$ Pharmacol Exp Ther 348:465-477.

Egger G, Liang G, Aparicio A, and Jones PA (2004) Epigenetics in human disease and prospects for epigenetic therapy. Nature 429:457-463.

Ehmer U, Kalthoff S, Lankisch TO, Freiberg N, Manns MP, and Strassburg CP (2010) Shared regulation of UGT1A7 by hepatocyte nuclear factor (HNF) $1 \alpha$ and HNF4 $\alpha$. Drug Metab Dispos 38:1246-1257.

Gagnon JF, Bernard O, Villeneuve L, Tetu B, and Guillemette C (2006) Irinotecan inactivation is modulated by epigenetic silencing of UGT1A1 in colon cancer. Clin Cancer Res 12:1850-1858. Gardner-Stephen DA and Mackenzie PI (2007) Isolation of the UDP-glucuronosyltransferase 1A3 and $1 \mathrm{~A} 4$ proximal promoters and characterization of their dependence on the transcription factor hepatocyte nuclear factor $1 \alpha$. Drug Metab Dispos 35:116-120.

Gregory PA, Lewinsky RH, Gardner-Stephen DA, and Mackenzie PI (2004) Coordinate regulation of the human UDP-glucuronosyltransferase 1A8, 1A9, and 1A10 genes by hepatocyte nuclear factor 1alpha and the caudal-related homeodomain protein 2. Mol Pharmacol 65:953-963.

He H, Nie YL, Li JF, Meng XG, Yang WH, Chen YL, Wang SJ, Ma X, Kan QC, and Zhang LR (2016) Developmental regulation of CYP3A4 and CYP3A7 in Chinese Han population. Drug Metab Pharmacokinet 31:433-444.

Ingelman-Sundberg M, Zhong XB, Hankinson O, Beedanagari S, Yu AM, Peng L, and Osawa Y (2013) Potential role of epigenetic mechanisms in the regulation of drug metabolism and transport. Drug Metab Dispos 41:1725-1731.

Ishii Y, Hansen AJ, and Mackenzie PI (2000) Octamer transcription factor-1 enhances hepatic nuclear factor-1alpha-mediated activation of the human UDP glucuronosyltransferase 2B7 promoter. Mol Pharmacol 57:940-947.

Ivanov M, Barragan I, and Ingelman-Sundberg M (2014) Epigenetic mechanisms of importance for drug treatment. Trends Pharmacol Sci 35:384-396.

Jaenisch R and Bird A (2003) Epigenetic regulation of gene expression: how the genome integrates intrinsic and environmental signals. Nat Genet 33 (Suppl):245-254.

Kanou M, Usui T, Ueyama H, Sato H, Ohkubo I, and Mizutani T (2004) Stimulation of transcriptional expression of human UDP-glucuronosyltransferase 1A1 by dexamethasone. Mol Biol Rep 31:151-158.

Kawade N and Onishi S (1981) The prenatal and postnatal development of UDPglucuronyltransferase activity towards bilirubin and the effect of premature birth on this activity in the human liver. Biochem $J$ 196:257-260.

Kim IW, Han N, Burckart GJ, and Oh JM (2014) Epigenetic changes in gene expression for drugmetabolizing enzymes and transporters. Pharmacotherapy 34:140-150.

Li Y, Buckley D, Wang S, Klaassen CD, and Zhong XB (2009a) Genetic polymorphisms in the TATA box and upstream phenobarbital-responsive enhancer module of the UGT1A1 promoter have combined effects on UDP-glucuronosyltransferase $1 \mathrm{~A} 1$ transcription mediated by constitutive androstane receptor, pregnane $\mathrm{X}$ receptor, or glucocorticoid receptor in human liver. Drug Metab Dispos 37:1978-1986.

Li Y, Cui Y, Hart SN, Klaassen CD, and Zhong XB (2009b) Dynamic patterns of histone methylation are associated with ontogenic expression of the Cyp3a genes during mouse liver maturation. Mol Pharmacol 75:1171-1179.

Lund AH and van Lohuizen M (2004) Polycomb complexes and silencing mechanisms. Curr Opin Cell Biol 16:239-246.

Mahajan MA and Samuels HH (2008) Nuclear receptor coactivator/coregulator NCoA6(NRC) is a pleiotropic coregulator involved in transcription, cell survival, growth and development. Nucl Recept Signal 6:e02.

Miyagi SJ and Collier AC (2011) The development of UDP-glucuronosyltransferases 1A1 and 1A6 in the pediatric liver. Drug Metab Dispos 39:912-919.

Nie YL, He H, Li JF, Meng XG, Yan L, Wang P, Wang SJ, Bi HZ, Zhang LR, and Kan QC (2017) Hepatic expression of transcription factors affecting developmental regulation of UGT1A1 in the Han Chinese population. Eur J Clin Pharmacol 73:29-37.

Oda S, Fukami T, Yokoi T, and Nakajima M (2013) Epigenetic regulation is a crucial factor in the repression of UGT1A1 expression in the human kidney. Drug Metab Dispos 41:1738-1743.

Odom DT, Zizlsperger N, Gordon DB, Bell GW, Rinaldi NJ, Murray HL, Volkert TL, Schreiber J, Rolfe PA, Gifford DK, et al. (2004) Control of pancreas and liver gene expression by HNF transcription factors. Science 303:1378-1381.

Otte AP and Kwaks TH (2003) Gene repression by polycomb group protein complexes: a distinct complex for every occasion? Curr Opin Genet Dev 13:448-454.

Peng L and Zhong X (2015) Epigenetic regulation of drug metabolism and transport. Acta Pharm $\operatorname{Sin} B$ 5:106-112.

Ramírez J, Mirkov S, Zhang W, Chen P, Das S, Liu W, Ratain MJ, and Innocenti F (2008) Hepatocyte nuclear factor-1 alpha is associated with UGT1A1, UGT1A9 and UGT2B7 mRNA expression in human liver. Pharmacogenomics $J$ 8:152-161.

Rickels R, Hu D, Collings CK, Woodfin AR, Piunti A, Mohan M, Herz HM, Kvon E, and Shilatifard A (2016) An evolutionary conserved epigenetic mark of polycomb response elements implemented by Trx/MLL/COMPASS. Mol Cell 63:318-328.

Rosenfeld MG, Lunyak VV, and Glass CK (2006) Sensors and signals: a coactivator/corepressor/epigenetic code for integrating signal-dependent programs of transcriptional response. Genes Dev 20:1405-1428.

Sasaki H and Matsui Y (2008) Epigenetic events in mammalian germ-cell development: reprogramming and beyond. Nat Rev Genet 9:129-140.

Sharma V, Malgulwar PB, Purkait S, Patil V, Pathak P, Agrawal R, Kulshreshtha R, Mallick S, Julka PK, Suri A, et al. (2017) Genome-wide ChIP-seq analysis of EZH2-mediated H3K27me3 
target gene profile highlights differences between low- and high-grade astrocytic tumors. Carcinogenesis 38:152-161.

Shi X, Hong T, Walter KL, Ewalt M, Michishita E, Hung T, Carney D, Peña P, Lan F, Kaadige MR, et al. (2006) ING2 PHD domain links histone H3 lysine 4 methylation to active gene repression. Nature 442:96-99.

Shi X, Kachirskaia I, Walter KL, Kuo JH, Lake A, Davrazou F, Chan SM, Martin DG, Fingerman IM, Briggs SD, et al. (2007) Proteome-wide analysis in Saccharomyces cerevisiae identifies several PHD fingers as novel direct and selective binding modules of histone $\mathrm{H} 3$ methylated at either lysine 4 or lysine 36. J Biol Chem 282:2450-2455.

Sun J, Zhao Y, McGreal R, Cohen-Tayar Y, Rockowitz S, Wilczek C, Ashery-Padan R, Shechter D, Zheng D, and Cvekl A (2016) Pax6 associates with H3K4-specific histone methyltransferase Mll1, Mll2, and Set1a and regulates $\mathrm{H} 3 \mathrm{~K} 4$ methylation at promoters and enhancers. Epigenetics Chromatin 9:37.

Surapureddi S, Rana R, and Goldstein JA (2011) NCOA6 differentially regulates the expression of the CYP2C9 and CYP3A4 genes. Pharmacol Res 63:405-413.

Surapureddi S, Rana R, Reddy JK, and Goldstein JA (2008) Nuclear receptor coactivator 6 mediates the synergistic activation of human cytochrome P-450 2C9 by the constitutive androstane receptor and hepatic nuclear factor-4alpha. Mol Pharmacol 74:913-923.

Toide K, Takahashi Y, Yamazaki H, Terauchi Y, Fujii T, Parkinson A, and Kamataki T (2002) Hepatocyte nuclear factor-1alpha is a causal factor responsible for interindividual differences in the expression of UDP-glucuronosyltransferase 2B7 mRNA in human livers. Drug Metab Dispos 30:613-615.
Wysocka J, Swigut T, Xiao H, Milne TA, Kwon SY, Landry J, Kauer M, Tackett AJ, Chait BT, Badenhorst P, et al. (2006) A PHD finger of NURF couples histone H3 lysine 4 trimethylation with chromatin remodelling. Nature 442:86-90.

Xiao B, Jing C, Wilson JR, Walker PA, Vasisht N, Kelly G, Howell S, Taylor IA, Blackburn GM, and Gamblin SJ (2003) Structure and catalytic mechanism of the human histone methyltransferase SET7/9. Nature 421:652-656.

Yan L, Wang Y, Liu J, Nie Y, Zhong XB, Kan Q, and Zhang L (2017) Alterations of histone modifications contribute to pregnane $\mathrm{X}$ receptor-mediated induction of CYP3A4 by rifampicin. Mol Pharmacol 92:113-123.

Yasar U, Greenblatt DJ, Guillemette C, and Court MH (2013) Evidence for regulation of UDPglucuronosyltransferase (UGT) 1A1 protein expression and activity via DNA methylation in healthy human livers. $J$ Pharm Pharmacol 65:874-883.

Zanger UM, Klein K, Thomas M, Rieger JK, Tremmel R, Kandel BA, Klein M, and Magdy T (2014) Genetics, epigenetics, and regulation of drug-metabolizing cytochrome p450 enzymes. Clin Pharmacol Ther 95:258-261.

Address correspondence to: Dr. Li-Rong Zhang, Department of Pharmacology, School of Basic Medicine, Zhengzhou University, 100 Science Road Zhengzhou 450001, People's Republic of China. E-mail: zhanglirongzzu@126.com 